\title{
Common Data Elements for Neurological Registries
}

\author{
Janet Warner ${ }^{1}$, Megan Johnston ${ }^{1}$, Lawrence Korngut ${ }^{1}$, Nathalie Jette ${ }^{1,2}$, \\ Tamara Pringsheim ${ }^{l}$
}

Can J Neurol Sci. 2013; 40: Suppl. 2 - S62-S63

At the outset of this project, one aim was to define a set of core data fields to include in all neurological registries in Canada. This project targeted neurological registries in Canada for all priority neurological conditions identified in the call for proposal including: Alzheimer's disease (AD) and other dementias, amyotrophic lateral sclerosis (ALS), brain tumours, cerebral palsy (CP), dystonia, epilepsy, Huntington's disease (HD), hydrocephalus, multiple sclerosis (MS), muscular dystrophy (MD), Parkinson's disease (PD), spina bifida, spinal cord injuries (SCI), Tourette syndrome and traumatic brain injury (TBI). The project team sought to identify all existing neurological condition registries in Canada to consult as stakeholders in this project. Disease experts from all of the above priority conditions were included in the process. Neurological registries represented in the project included:

- The Canadian Cerebral Palsy Registry - a voluntary registry including children with $\mathrm{CP}$ across several jurisdictions in Canada. (Edmonton, Alberta).

- The Canadian Neuromuscular Disease Registry (CNDR) - a voluntary registry which includes MD, ALS and all other adults and children with neuromuscular disease in Canada (Calgary, Alberta, www.cndr.org)

- Hydrocephalus Registry (under development)

- The North American Research Committee on Multiple Sclerosis (NARCOMS) Registry - a voluntary online registry for patients worldwide with MS (Birmingham, Alabama, www.narcoms.org)

- The Ontario Stroke Registry - formerly known as the Registry of the Canadian Stroke Network, a mandatory registry under Ontario's Personal Health Information Protection Act (PHIPA) based at the Institute for Clinical Evaluative Sciences (ICES) in Toronto, Ontario. http://www.ices.on.ca/webpage .cfm?site_id=1\&org_id=26\&morg_id=0\&gsec_id=7071\&item_ $\mathrm{id}=7071$

- The Quebec Myotonic Dystrophy Registry - a voluntary registry for Quebec patients with myotonic dystrophy (a form of MD) (Quebec, Quebec http://www.dystrophiemyotonique.chuq. qc.ca/ENG/registry-why.html)

- The Rick Hansen Spinal Cord Injury Registry (RHSCIR) - a voluntary registry which includes individuals with SCI from across Canada (Vancouver, British Columbia, www.rickhansen registry.org)
- The Southern Alberta Dementia Registry (under development)

- The Sudden Unexplained Death in Epilepsy (SUDEP) Registry (under development)

- The Tourette syndrome International Consortium (TIC) Database - an international database of Tourette's patients from 27 countries housed at the BC Children's Hospital in Vancouver, British Columbia.

\section{BACKGROUND}

In selecting elements for a registry, several factors must be considered:

- Importance of the elements for the integrity of the registry

- Reliability of data collection in each element

- Necessity for analysis of the primary outcome of the registry

- Burden of data collection in each element (time and cost)

Data element selection can be simplified if clinical data standards exist for the disease or condition of interest. Utilizing data elements that adhere to clinical standards can facilitate comparisons across registries; improve efficiency during the establishment of registries; promote effective sharing and data linkage between registries; and can help to ensure the meaning of information collected by different registries is the same.

We identified a number of potential sources of core data fields available worldwide including:

1) National Institute of Neurological Disorders and Stroke (NINDS) based at the National Institutes of Health in the United States maintains a list of common data elements for 8 of the priority neurological conditions including ALS, PD, HD, MD, epilepsy, SCI, MS, and TBI.

2) Ontario Brain Institute based in Ontario Canada is preparing common data elements for CP, and epilepsy.

3) Translational Research in Europe for the Assessment and Treatment of Neuromuscular Disease (TREAT-NMD) has prepared common data elements for number of neuromuscular conditions including Duchenne, congenital, and myotonic muscular dystrophies.

4) The EURO-MOTOR project in Europe is defining common data elements for ALS databases in Europe.

From the ${ }^{1}$ Hotchkiss Brain Institute, University of Calgary, Calgary, Alberta; ${ }^{2}$ Institute of Public Health, University of Calgary, Calgary, Alberta.

Final Revisions Submitted JanUary 28, 2013.

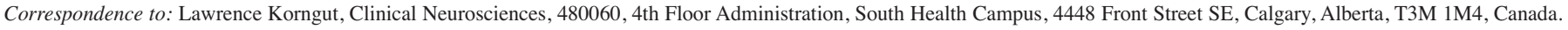
Email: Lawrence.korngut@albertahealthservices.ca. 
5) The Patient-Reported Outcomes Measurement Information System (PROMIS) project created a web-based resource that features data field banks, case report form banks, and centralized access to computerized-adaptive testing for some measures.

6) The EPIRARE project based in Europe has been discussing common data elements for rare disease registries in Europe and globally.

Overall, inclusion of core data elements in registries can enhance registry feasibility and sustainability by providing the opportunity for sharing of data between registries in a meaningful way.

\section{METHOD}

In May 2012, the project team held its first team meeting and initial discussion around core data fields occurred. At that meeting a brief review of potential items based on the above identified data sources was presented. Consensus at the meeting was to hold a Delphi method consultation among all investigators and stakeholders to identify potential common data elements.

Over the summer, the Delphi method consultation was configured and held using the web-based survey platform Survey Gizmo (Boulder CO, www.surveygizmo.com). Thirty-one people received the survey and there was a $71 \%$ completion rate.

The survey featured two questions regarding each proposed data element:

1) Should the item be collected from registry participants?

2) Is the proposed field for collecting the information from participants appropriate?

Participants could respond on a radio button scale featuring the options Strongly Disagree, Disagree, Neutral, Agree, Strongly Agree, and No Opinion.

In September 2012, the results of the survey were reviewed the second project team meeting and final discussion around the proposed core data fields occurred.

\section{Discussion}

In general, the results of the Delphi method consultation did not provide clarity on core data elements for neurological registries in Canada. The registry team decided at the September meeting that very few elements could be recommended based on the results of the Delphi consultation. Additionally, substantial challenges in collecting any elements across the entire neurological disease spectrum were identified due to the heterogeneity of the diseases/conditions being considered and the relevant clinical measures and outcomes especially when considering the spectrum of the diseases across pediatric and adult audiences. The mobility of patients between regions within Canada was identified as being a major concern due to the lack of a consistent identifier apart from a Social Insurance Number (SIN). Focus group data collected in the spring of 2012 indicated that patients were highly unwilling to share their SIN number for registry purposes. There were also substantial concerns regarding a duplication of effort against the other identified organizations creating common data elements within some of the disease groups and a considerable issue as to how to address adequate stewardship of the dataset beyond the scope of this project.

\section{RECOMMENDATIONS}

The project team recommended that neurological core data elements are essential and should be developed but the process required is beyond the capability of the current project. Core data elements will require a national group of registry leaders and experts to provide oversight and updating of core data elements to ensure validity and relevance over time.

The project team arrived at the consensus recommendation that neurological disease registries with patient contact in Canada should collect the following elements to maximize the compatibility of data between registries and prevent overlap.
$\checkmark$ Full legal name
$\checkmark$ Date of birth
$\checkmark$ Place of birth
$\checkmark$ Sex
$\checkmark$ Disease/Diagnosis
$\checkmark$ Provincial Health Number (if required for data linkage based on registry needs)

It should be noted that the above elements incorporate the participant's full legal name, date of birth and place of birth as the sole "unique" identifier for a registry participant. Provincial health numbers are not considered a unique identifier as they change from province to province if a registry participant moves. This is important for multi-jurisdictional registries to avoid having a patient who moves provinces registered twice.

Gender, date of birth, and provincial health number will be required if the registry desires linkage with administrative data. For registries with the sole purpose of linking to administrative data it may not be possible to ethically justify the collection of the legal name.

The project team also recommended that registries examine relevant core data elements as previously identified from international and other applicable groups. It is recommended that registries be willing to share case report forms to enhance the ability to collaborate with new and existing registries.

Finally, it is recommended that registries designing case report forms consider validated sources of questions available in Canada such as the Canadian Community Health Survey (CCHS) and other Statistics Canada surveys. 